IJMMS 31:3 (2002) 167-175

PII. S0161171202109288

http://ijmms.hindawi.com

(c) Hindawi Publishing Corp.

\title{
CR-SUBMANIFOLDS OF A NEARLY TRANS-SASAKIAN MANIFOLD
}

\author{
FALLEH R. AL-SOLAMY
}

Received 26 September 2001

This paper considers the study of CR-submanifolds of a nearly trans-Sasakian manifold, generalizing the results of trans-Sasakian manifolds and thus those of Sasakian manifolds.

2000 Mathematics Subject Classification: 53C40.

1. Introduction. In 1978, Bejancu introduced the notion of CR-submanifold of a Kaehler manifold [1]. Since then several papers on CR-submanifolds of Kaehler manifold have been published. On the other hand, CR-submanifolds of a Sasakian manifold have been studied by Kobayashi [6], Shahid et al. [10], Yano and Kon [11], and others. Bejancu and Papaghuic [2] studied CR-submanifolds of a Kenmotsu manifold. In 1985, Oubina introduced a new class of almost contact metric manifold known as transSasakian manifold [7]. This class contains $\alpha$-Sasakian and $\beta$-Kenmotsu manifold [5]. Geometry of CR-submanifold of trans-Sasakian manifold was studied by Shahid [8, 9]. A nearly trans-Sasakian manifold [4] is a more general concept.

The results of this paper are the generalization of the results obtained earlier by several authors, namely $[6,8,9]$ and others.

2. Preliminaries. Let $\bar{M}$ be an $n$-dimensional almost contact metric manifold with an almost contact metric structure $(\phi, \xi, \eta, g)$ satisfying [3]

$$
\begin{gathered}
\phi^{2} X=-X+\eta(X) \xi, \quad \eta(\xi)=1, \phi \circ \xi=0, \\
g(\phi X, \phi Y)=g(X, Y)-\eta(X) \eta(Y), \quad g(X, \xi)=\eta(X),
\end{gathered}
$$

where $X$ and $Y$ are vector fields tangent to $\bar{M}$.

An almost contact metric structure $(\phi, \xi, \eta, g)$ on $\bar{M}$ is called trans-Sasakian if [7]

$$
\left(\bar{\nabla}_{X} \phi\right)(Y)=\alpha\{g(X, Y) \xi-\eta(Y) X\}+\beta\{g(\phi X, Y) \xi-\eta(Y) \phi X\},
$$

where $\alpha$ and $\beta$ are nonzero constants, $\bar{\nabla}$ denotes the Riemannian connection of $g$ on $\bar{M}$, and we say that the trans-Sasakian structure is of type $(\alpha, \beta)$.

Further, an almost contact metric manifold $\bar{M}(\phi, \xi, \eta, g)$ is called nearly transSasakian if [4]

$$
\begin{aligned}
\left(\bar{\nabla}_{X} \phi\right)(Y)+\left(\bar{\nabla}_{Y} \phi\right)(X)= & \alpha\{2 g(X, Y) \xi-\eta(Y) X-\eta(X) Y\} \\
& -\beta\{\eta(Y) \phi X+\eta(X) \phi Y\} .
\end{aligned}
$$

It is clear that any trans-Sasakian manifold, and thus any Sasakian manifold, satisfies the above relation. 
Let $M$ be an $m$-dimensional isometrically immersed submanifold of a nearly transSasakian manifold $\bar{M}$ and denote by the same $g$ the Riemannian metric tensor field induced on $M$ from that of $\bar{M}$.

DEFINITION 2.1. An $m$-dimensional Riemannian submanifold $M$ of a nearly transSasakian manifold $\bar{M}$ is called a CR-submanifold if $\xi$ is tangent to $M$ and there exists a differentiable distribution $D: x \in M \rightarrow D_{x} \subset T_{x} M$ such that

(1) the distribution $D_{x}$ is invariant under $\phi$, that is, $\phi D_{x} \subset D_{x}$ for each $x \in M$;

(2) the complementary orthogonal distribution $D^{\perp}: x \in M \rightarrow D_{x}^{\perp} \subset T_{x} M$ of $D$ is anti-invariant under $\phi$, that is, $\phi D_{x}^{\perp} \subset T_{x}^{\perp} M$ for all $x \in M$, where $T_{x} M$ and $T_{x}^{\perp} M$ are the tangent space and the normal space of $M$ at $x$, respectively.

If $\operatorname{dim} D_{x}^{\perp}=0$ (resp., $\operatorname{dim} D_{x}=0$ ), then the CR-submanifold is called an invariant (resp., anti-invariant) submanifold. The distribution $D$ (resp., $D^{\perp}$ ) is called the horizontal (resp., vertical) distribution. Also, the pair $\left(D, D^{\perp}\right)$ is called $\xi$-horizontal (resp., vertical) if $\xi_{x} \in D_{x}$ (resp., $\xi_{x} \in D_{x}^{\perp}$ ) [6].

For any vector field $X$ tangent to $M$, we put [6]

$$
X=P X+Q X,
$$

where $P X$ and $Q X$ belong to the distribution $D$ and $D^{\perp}$.

For any vector field $N$ normal to $M$, we put [6]

$$
\phi N=B N+C N,
$$

where $B N$ (resp., $C N$ ) denotes the tangential (resp., normal) component of $\phi N$.

Let $\bar{\nabla}$ (resp., $\nabla$ ) be the covariant differentiation with respect to the Levi-Civita connection on $\bar{M}$ (resp., $M$ ). The Gauss and Weingarten formulas for $M$ are respectively given by

$$
\bar{\nabla}_{X} Y=\nabla_{X} Y+h(X, Y) ; \quad \bar{\nabla}_{X} N=-A_{N} X+\nabla_{X}^{\perp} N,
$$

for $X, Y \in T M$ and $N \in T^{\perp} M$, where $h$ (resp., $A$ ) is the second fundamental form (resp., tensor) of $M$ in $\bar{M}$, and $\nabla^{\perp}$ denotes the normal connection. Moreover, we have

$$
g(h(X, Y), N)=g\left(A_{N} X, Y\right) .
$$

3. Some basic lemmas. First we prove the following lemma.

LEMMA 3.1. Let $M$ be a CR-submanifold of a nearly trans-Sasakian manifold $\bar{M}$. Then

$$
\begin{gathered}
P\left(\nabla_{X} \phi P Y\right)+P\left(\nabla_{Y} \phi P X\right)-P\left(A_{\phi Q X} Y\right)-P\left(A_{\phi Q Y} X\right) \\
=\phi P \nabla_{X} Y+\phi P \nabla_{Y} X+2 \alpha g(X, Y) P \xi-\alpha \eta(Y) P X \\
-\alpha \eta(X) P Y-\beta \eta(Y) \phi P X+\beta \eta(X) \phi P Y, \\
Q\left(\nabla_{X} \phi P Y\right)+Q\left(\nabla_{Y} \phi P X\right)-Q\left(A_{\phi Q X} Y\right)-Q\left(A_{\phi Q Y} X\right) \\
=2 B h(X, Y)-\alpha \eta(Y) Q X-\alpha \eta(X) Q Y+2 \alpha g(X, Y) Q \xi \\
h(X, \phi P Y)+h(Y, \phi P X)+\nabla_{X}^{\frac{1}{X}} \phi Q Y+\nabla_{Y}^{\perp} \phi Q X \\
=\phi Q \nabla_{Y} X+\phi Q \nabla_{X} Y+2 C h(X, Y)-\beta \eta(Y) \phi Q X-\beta \eta(X) \phi Q Y
\end{gathered}
$$

for $X, Y \in T M$. 
Proof. From the definition of the nearly trans-Sasakian manifold and using (2.4), (2.5), and (2.6), we get

$$
\begin{aligned}
\nabla_{X} \phi P Y+ & h(X, \phi P Y)-A_{\phi Q Y} X+\nabla_{X}^{\perp} \phi Q Y-\phi\left(\nabla_{X} Y+h(X, Y)\right) \\
& +\nabla_{Y} \phi P X+h(Y, \phi P X)-A_{\phi Q X} Y+\nabla_{Y}^{\perp} \phi Q X-\phi\left(\nabla_{Y} X+h(X, Y)\right) \\
= & \alpha\{2 g(X, Y) \xi-\eta(Y) X-\eta(X) Y\}-\beta\{\eta(Y) \phi X+\eta(X) \phi Y\}
\end{aligned}
$$

for any $X, Y \in T M$.

Now using (2.4) and equaling horizontal, vertical, and normal components in (3.4), we get the result.

LEMMA 3.2. Let $M$ be a CR-submanifold of a nearly trans-Sasakian manifold $\bar{M}$. Then

$$
\begin{aligned}
2\left(\bar{\nabla}_{X} \phi\right)(Y)= & \nabla_{X} \phi Y-\nabla_{Y} \phi X+h(X, \phi Y)-h(Y, \phi X)-\phi[X, Y] \\
& +\alpha\{2 g(X, Y) \xi-\eta(Y) X-\eta(X) Y\}-\beta\{\eta(Y) \phi X+\eta(X) \phi Y\}
\end{aligned}
$$

for any $X, Y \in D$.

Proof. By Gauss formula (2.6), we get

$$
\bar{\nabla}_{X} \phi Y-\bar{\nabla}_{Y} \phi X=\nabla_{X} \phi Y+h(X, \phi Y)-\nabla_{Y} \phi X-h(Y, \phi X) .
$$

Also, we have

$$
\bar{\nabla}_{X} \phi Y-\bar{\nabla}_{Y} \phi X=\left(\bar{\nabla}_{X} \phi\right)(Y)-\left(\bar{\nabla}_{Y} \phi\right)(X)+\phi[X, Y]
$$

From (3.6) and (3.7), we get

$$
\left(\bar{\nabla}_{X} \phi\right)(Y)-\left(\bar{\nabla}_{Y} \phi\right)(X)=\nabla_{X} \phi Y+h(X, \phi Y)-\nabla_{Y} \phi X-h(Y, \phi X)-\phi[X, Y] .
$$

Also for nearly trans-Sasakian manifolds, we have

$$
\left(\bar{\nabla}_{X} \phi\right)(Y)+\left(\bar{\nabla}_{Y} \phi\right)(X)=\alpha\{2 g(X, Y) \xi-\eta(Y) X-\eta(X) Y\}-\beta\{\eta(Y) \phi X+\eta(X) \phi Y\} .
$$

Combining (3.8) and (3.9), the lemma follows.

In particular, we have the following corollary.

COROLLARY 3.3. Let $M$ be a $\xi$-vertical CR-submanifold of a nearly trans-Sasakian manifold, then

$$
2\left(\bar{\nabla}_{X} \phi\right)(Y)=\nabla_{X} \phi Y-\nabla_{Y} \phi X+h(X, \phi Y)-h(Y, \phi X)-\phi[X, Y]+2 \alpha g(X, Y) \xi
$$

for any $X, Y \in D$.

Similarly, by Weingarten formula (2.6), we get the following lemma. 
LEMMA 3.4. Let $M$ be a CR-submanifold of a nearly trans-Sasakian manifold $\bar{M}$, then

$$
\begin{aligned}
2\left(\bar{\nabla}_{Y} \phi\right)(Z)= & A_{\phi Y} Z-A_{\phi Z} Y+\nabla_{Y}^{\perp} \phi Z-\nabla_{Z}^{\frac{1}{Z}} \phi Y-\phi[Y, Z] \\
& +\alpha\{2 g(Y, Z) \xi-\eta(Y) Z-\eta(Z) Y\}-\beta\{\eta(Y) \phi Z+\eta(Z) \phi Y\}
\end{aligned}
$$

for any $Y, Z \in D^{\perp}$.

COROLLARY 3.5. Let M be a $\xi$-horizontal CR-submanifold of a nearly trans-Sasakian manifold, then

$$
2\left(\bar{\nabla}_{Y} \phi\right)(Z)=A_{\phi Y} Z-A_{\phi Z} Y+\nabla_{Y}^{\frac{1}{Y}} \phi Z-\nabla_{Z}^{\frac{1}{Z}} \phi Y-\phi[Y, Z]+2 \alpha g(Y, Z) \xi
$$

for any $Y, Z \in D^{\perp}$.

LEMMA 3.6. Let $M$ be a CR-submanifold of a nearly trans-Sasakian manifold $\bar{M}$, then

$$
\begin{aligned}
2\left(\bar{\nabla}_{X} \phi\right)(Y)= & \alpha\{2 g(X, Y) \xi-\eta(Y) X-\eta(X) Y\}-\beta\{\eta(Y) \phi X+\eta(X) \phi Y\} \\
& -A_{\phi Y} X+\nabla_{X}^{\perp} \phi Y-\nabla_{Y} \phi X-h(Y, \phi X)-\phi[X, Y]
\end{aligned}
$$

for any $X \in D$ and $Y \in D^{\perp}$.

\section{Parallel distributions}

Definition 4.1. The horizontal (resp., vertical) distribution $D$ (resp., $D^{\perp}$ ) is said to be parallel [1] with respect to the connection $\nabla$ on $M$ if $\nabla_{X} Y \in D$ (resp., $\nabla_{Z} W \in D^{\perp}$ ) for any vector field $X, Y \in D$ (resp., $W, Z \in D^{\perp}$ ).

Now we prove the following proposition.

Proposition 4.2. Let M be a $\xi$-vertical CR-submanifold of a nearly trans-Sasakian manifold $\bar{M}$. If the horizontal distribution $D$ is parallel, then

$$
h(X, \phi Y)=h(Y, \phi X)
$$

for all $X, Y \in D$.

Proof. Using parallelism of horizontal distribution $D$, we have

$$
\nabla_{X} \phi Y \in D, \quad \nabla_{Y} \phi X \in D \quad \text { for any } X, Y \in D .
$$

Thus using the fact that $Q X=Q Y=0$ for $Y \in D$, (3.2) gives

$$
B h(X, Y)=g(X, Y) Q \xi \quad \text { for any } X, Y \in D .
$$

Also, since

$$
\phi h(X, Y)=B h(X, Y)+C h(X, Y)
$$

then

$$
\phi h(X, Y)=g(X, Y) Q \xi+C h(X, Y) \quad \text { for any } X, Y \in D .
$$

Next from (3.3), we have

$$
h(X, \phi Y)+h(Y, \phi X)=2 C h(X, Y)=2 \phi h(X, Y)-2 g(X, Y) Q \xi,
$$


for any $X, Y \in D$. Putting $X=\phi X \in D$ in (4.6), we get

$$
h(\phi X, \phi Y)+h\left(Y, \phi^{2} X\right)=2 \phi h(\phi X, Y)-2 g(\phi X, Y) Q \xi
$$

or

$$
h(\phi X, \phi Y)-h(Y, X)=2 \phi h(\phi X, Y)-2 g(\phi X, Y) Q \xi .
$$

Similarly, putting $Y=\phi Y \in D$ in (4.6), we get

$$
h(\phi Y, \phi X)-h(X, Y)=2 \phi h(X, \phi Y)-2 g(X, \phi Y) Q \xi .
$$

Hence from (4.8) and (4.9), we have

$$
\phi h(X, \phi Y)-\phi h(Y, \phi X)=g(X, \phi Y) Q \xi-g(\phi X, Y) Q \xi .
$$

Operating $\phi$ on both sides of (4.10) and using $\phi \xi=0$, we get

$$
h(X, \phi Y)=h(Y, \phi X)
$$

for all $X, Y \in D$.

Now, for the distribution $D^{\perp}$, we prove the following proposition.

Proposition 4.3. Let $M$ be a $\xi$-vertical CR-submanifold of a nearly trans-Sasakian manifold $\bar{M}$. If the distribution $D^{\perp}$ is parallel with respect to the connection on $M$, then

$$
\left(A_{\phi Y} Z+A_{\phi Z} Y\right) \in D^{\perp} \quad \text { for any } Y, Z \in D^{\perp} .
$$

Proof. Let $Y, Z \in D^{\perp}$, then using Gauss and Weingarten formula (2.6), we obtain

$$
\begin{aligned}
&-A_{\phi Z} Y+\nabla_{\frac{1}{Y}}^{\perp} \phi Z-A_{\phi Y} Z+\nabla_{Z}^{\frac{1}{Z}} \phi Y \\
&= \phi \nabla_{Y} Z+\phi \nabla_{Z} Y+2 \phi h(Y, Z) \\
&+\alpha\{2 g(X, Y) \xi-\eta(Y) Z-\eta(Z) Y\}-\beta\{\eta(Y) \phi Z+\eta(Z) \phi Y\}
\end{aligned}
$$

for any $Y, Z \in D^{\perp}$. Taking inner product with $X \in D$ in (4.13), we get

$$
g\left(A_{\phi Y} Z, X\right)+g\left(A_{\phi Z} Y, X\right)=g\left(\nabla_{Y} Z, \phi X\right)+g\left(\nabla_{Z} Y, \phi X\right) .
$$

If the distribution $D^{\perp}$ is parallel, then $\nabla_{Y} Z \in D^{\perp}$ and $\nabla_{Z} Y \in D^{\perp}$ for any $Y, Z \in D^{\perp}$. So from (4.14) we get

$$
g\left(A_{\phi Y} Z, X\right)+g\left(A_{\phi Z} Y, X\right)=0 \quad \text { or } \quad g\left(A_{\phi Y} Z+A_{\phi Z} Y, X\right)=0
$$

which is equivalent to

$$
\left(A_{\phi Y} Z+A_{\phi Z} Y\right) \in D^{\perp} \text { for any } Y, Z \in D^{\perp},
$$

and this completes the proof.

Definition 4.4 [6]. A CR-submanifold is said to be mixed totally geodesic if $h(X, Z)=0$ for all $X \in D$ and $Z \in D^{\perp}$. 
The following lemma is an easy consequence of (2.7).

LEMMA 4.5. Let $M$ be a CR-submanifold of a nearly trans-Sasakian manifold $\bar{M}$. Then $M$ is mixed totally geodesic if and only if $A_{N} X \in D$ for all $X \in D$.

Definition 4.6 [6]. A normal vector field $N \neq 0$ is called $D$-parallel normal section if $\nabla_{X}^{+} N=0$ for all $X \in D$.

Now we have the following proposition.

Proposition 4.7. Let $M$ be a mixed totally geodesic $\xi$-vertical CR-submanifold of a nearly trans-Sasakian manifold $\bar{M}$. Then the normal section $N \in \phi D^{\perp}$ is $D$-parallel if and only if $\nabla_{X} \phi N \in D$ for all $X \in D$.

Proof. Let $N \in \phi D^{\perp}$. Then from (3.2) we have

$$
Q\left(\nabla_{Y} \phi X\right)=0 \text { for any } X \in D, Y \in D^{\perp} .
$$

In particular, we have $Q\left(\nabla_{Y} X\right)=0$. By using it in (3.3), we get

$$
\nabla_{X}^{\perp} \phi Q Y=\phi Q \nabla_{X} Y \quad \text { or } \quad \nabla_{X}^{\perp} N=-\phi Q \nabla_{X} \phi N .
$$

Thus, if the normal section $N \neq 0$ is $D$-parallel, then using Definition 4.6 and (4.18), we get

$$
\phi Q\left(\nabla_{X} \phi N\right)=0
$$

which is equivalent to $\nabla_{X} \phi N \in D$ for all $X \in D$. The converse part easily follows from (4.18).

5. Integrability conditions of distributions. First we calculate the Nijenhuis tensor $N_{\phi}(X, Y)$ on a nearly trans-Sasakian manifold $\bar{M}$. For this, first we prove the following lemma.

LEMMA 5.1. Let $\bar{M}$ be a nearly trans-Sasakian manifold, then

$$
\begin{aligned}
\left(\bar{\nabla}_{\phi X} \phi\right)(Y)= & 2 \alpha g(\phi X, Y) \xi-\eta(Y) \phi X+\beta \eta(Y) X-\beta \eta(X) \eta(Y) \xi \\
& -\eta(X) \bar{\nabla}_{Y} \xi+\phi\left(\bar{\nabla}_{Y} \phi\right)(X)+\eta\left(\bar{\nabla}_{Y} X\right) \xi
\end{aligned}
$$

for any $X, Y \in T \bar{M}$.

Proof. From the definition of nearly trans-Sasakian manifold $\bar{M}$, we have

$$
\begin{aligned}
\left(\bar{\nabla}_{\phi X} \phi\right)(Y)= & 2 \alpha g(\phi X, Y) \xi-\eta(Y) \phi X+\beta \eta(Y) X \\
& -\beta \eta(Y) \eta(X) \xi-\left(\bar{\nabla}_{Y} \phi\right)(\phi X) .
\end{aligned}
$$

Also, we have

$$
\begin{aligned}
\left(\bar{\nabla}_{Y} \phi\right)(\phi X) & =\bar{\nabla}_{Y} \phi^{2} X-\phi \bar{\nabla}_{Y} \phi X \\
& =\bar{\nabla}_{Y} \phi^{2} X-\phi \bar{\nabla}_{Y} \phi X+\phi\left(\phi \bar{\nabla}_{Y} X\right)-\phi\left(\phi \bar{\nabla}_{Y} X\right) \\
& =-\bar{\nabla}_{Y} X+\eta(X) \bar{\nabla}_{Y} \xi-\phi\left(\bar{\nabla}_{Y} \phi X-\phi \bar{\nabla}_{Y} X\right)-\phi\left(\phi \bar{\nabla}_{Y} X\right) \\
& =-\bar{\nabla}_{Y} X+\eta(X) \bar{\nabla}_{Y} \xi-\phi\left(\bar{\nabla}_{Y} \phi\right)(X)-\bar{\nabla}_{Y} X-\eta\left(\bar{\nabla}_{Y} X\right) \xi .
\end{aligned}
$$


Using (5.3) in (5.2), we get

$$
\begin{aligned}
\left(\bar{\nabla}_{\phi X} \phi\right)(Y)= & 2 \alpha g(\phi X, Y) \xi-\eta(Y) \phi X+\beta \eta(Y) X-\beta \eta(X) \eta(Y) \xi \\
& -\eta(X) \bar{\nabla}_{Y} \xi+\phi\left(\bar{\nabla}_{Y} \phi\right)(X)+\eta\left(\bar{\nabla}_{Y} X\right) \xi
\end{aligned}
$$

for any $X, Y \in T \bar{M}$, which completes the proof of the lemma.

On a nearly trans-Sasakian manifold $\bar{M}$, Nijenhuis tensor is given by

$$
N_{\phi}(X, Y)=\left(\bar{\nabla}_{\phi X} \phi\right)(Y)-\left(\bar{\nabla}_{\phi Y} \phi\right)(X)-\phi\left(\bar{\nabla}_{X} \phi\right)(Y)+\phi\left(\bar{\nabla}_{Y} \phi\right)(X)
$$

for any $X, Y \in T \bar{M}$.

From (5.1) and (5.5), we get

$$
\begin{aligned}
N_{\phi}(X, Y)= & 4 \alpha g(\phi X, Y) \xi-\eta(Y) \phi X+\eta(X) \phi Y+\beta \eta(Y) X \\
& -\beta \eta(X) Y-\eta(X) \bar{\nabla}_{Y} \xi+\eta(Y) \bar{\nabla}_{X} \xi+\eta\left(\bar{\nabla}_{Y} X\right) \xi \\
& -\eta\left(\bar{\nabla}_{X} Y\right) \xi+2 \phi\left(\bar{\nabla}_{Y} \phi\right)(X)-2 \phi\left(\bar{\nabla}_{X} \phi\right)(Y) .
\end{aligned}
$$

Thus using (2.3) in the above equation and after some calculations, we obtain

$$
\begin{aligned}
N_{\phi}(X, Y)= & 4 \alpha g(\phi X, Y) \xi+(2 \alpha-1) \eta(Y) \phi X+(2 \alpha+1) \eta(X) \phi Y-\beta \eta(Y) X \\
& -3 \beta \eta(X) Y+4 \beta \eta(X) \eta(Y) \xi-\eta(X) \bar{\nabla}_{Y} \xi+\eta(Y) \bar{\nabla}_{X} \xi \\
& +\eta\left(\bar{\nabla}_{Y} X\right) \xi-\eta\left(\bar{\nabla}_{X} Y\right) \xi+4 \phi\left(\bar{\nabla}_{Y} \phi\right)(X)
\end{aligned}
$$

for any $X, Y \in T \bar{M}$.

Now we prove the following proposition.

Proposition 5.2. Let $M$ be a $\xi$-vertical CR-submanifold of a nearly trans-Sasakian manifold $\bar{M}$. Then, the distribution $D$ is integrable if the following conditions are satisfied:

$$
S(X, Z) \in D, \quad h(X, \phi Z)=h(\phi X, Z)
$$

for any $X, Z \in D$.

Proof. The torsion tensor $S(X, Y)$ of the almost contact structure $(\phi, \xi, \eta, g)$ is given by

$$
S(X, Y)=N_{\phi}(X, Y)+2 d \eta(X, Y) \xi=N_{\phi}(X, Y)+2 g(\phi X, Y) \xi .
$$

Thus, we have

$$
S(X, Y)=[\phi X, \phi Y]-\phi[\phi X, Y]-\phi[X, \phi Y]+2 g(\phi X, Y) \xi
$$

for any $X, Y \in T M$.

Suppose that the distribution $D$ is integrable. So for $X, Y \in D, Q[X, Y]=0$ and $\eta([X, Y])=0$ as $\xi \in D^{\perp}$.

If $S(X, Y) \in D$, then from (5.7) and (5.9) we have

$\left\{2(2 \alpha+1) g(\phi X, Y) \xi+\eta([X, Y]) \xi+4\left(\phi \nabla_{Y} \phi X+\phi h(Y, \phi X)+Q \nabla_{Y} X+h(X, Y)\right)\right\} \in D$ 
or

$2(2 \alpha+1) g(\phi X, Y) Q \xi+\eta([X, Y]) Q \xi+4\left(\phi Q \nabla_{Y} \phi X+\phi h(Y, \phi X)+Q \nabla_{Y} X+h(X, Y)\right)=0$

for any $X, Y \in D$.

Replacing $Y$ by $\phi Z$ for $Z \in D$ in the above equation, we get

$2(2 \alpha+1) g(\phi X, \phi Z) Q \xi+4\left(\phi Q \nabla_{\phi Y} \phi X+\phi h(\phi Z, \phi X)+Q \nabla_{\phi Z} X+h(X, \phi Z)\right)=0$.

Interchanging $X$ and $Z$ for $X, Z \in D$ in (5.13) and subtracting these relations, we obtain

$$
\phi Q[\phi X, \phi Z]+Q[X, \phi Z]+h(X, \phi Z)-h(Z, \phi X)=0
$$

for any $X, Z \in D$ and the assertion follows.

Now, we prove the following proposition.

Proposition 5.3. Let $M$ be a CR-submanifold of a nearly trans-Sasakian manifold $\bar{M}$. Then

$$
A_{\phi Y} Z-A_{\phi Z} Y=\alpha(\eta(Y) Z-\eta(Z) Y)+\frac{1}{3} \phi P[Y, Z]
$$

for any $Y, Z \in D^{\perp}$.

Proof. For $Y, Z \in D^{\perp}$ and $X \in T(M)$, we get

$$
\begin{aligned}
2 g\left(A_{\phi Z} Y, X\right)= & 2 g(h(X, Y), \phi Z) \\
= & g(h(X, Y), \phi Z)+g(h(X, Y), \phi Z) \\
= & g\left(\bar{\nabla}_{X} Y, \phi Z\right)+g\left(\bar{\nabla}_{Y} X, \phi Z\right) \\
= & g\left(\bar{\nabla}_{X} Y+\bar{\nabla}_{Y} X, \phi Z\right) \\
= & -g\left(\phi\left(\bar{\nabla}_{X} Y+\bar{\nabla}_{Y} X\right), Z\right) \\
= & -g\left(\bar{\nabla}_{Y} \phi X+\bar{\nabla}_{X} \phi Y+\alpha \eta(X) Y+\alpha \eta(Y) X-2 \alpha g(X, Y) \xi, Z\right) \\
= & -g\left(\bar{\nabla}_{Y} \phi X, Z\right)-g\left(\bar{\nabla}_{X} \phi Y, Z\right)-\alpha \eta(X) g(Y, Z) \\
& -\alpha \eta(Y) g(X, Z)+2 \alpha \eta(Z) g(X, Y) \\
= & g\left(\bar{\nabla}_{Y} Z, \phi X\right)+g\left(A_{\phi Y} Z, X\right)-\alpha \eta(X) g(Y, Z) \\
& -\alpha \eta(Y) g(X, Z)+2 \alpha \eta(Z) g(X, Y) .
\end{aligned}
$$

The above equation is true for all $X \in T(M)$, therefore, transvecting the vector field $X$ both sides, we obtain

$$
2 A_{\phi Z} Y=A_{\phi Y} Z-\phi \bar{\nabla}_{Y} Z-\alpha g(Y, Z) \xi-\alpha \eta(Y) Z+2 \alpha \eta(Z) Y
$$

for any $Y, Z \in D^{\perp}$. Interchanging the vector fields $Y$ and $Z$, we get

$$
2 A_{\phi Y} Z=A_{\phi Z} Y-\phi \bar{\nabla}_{Z} Y-\alpha g(Y, Z) \xi-\alpha \eta(Z) Y+2 \alpha \eta(Y) Z .
$$

Subtracting (5.17) and (5.18), we get

$$
A_{\phi Y} Z-A_{\phi Z} Y=\alpha(\eta(Y) Z-\eta(Z) Y)+\frac{1}{3} \phi P[Y, Z]
$$

for any $Y, Z \in D^{\perp}$, which completes the proof. 
THEOREM 5.4. Let $M$ be a CR-submanifold of a nearly trans-Sasakian manifold $\bar{M}$. Then, the distribution $D^{\perp}$ is integrable if and only if

$$
A_{\phi Y} Z-A_{\phi Z} Y=\alpha(\eta(Y) Z-\eta(Z) Y), \quad \text { for any } Y, Z \in D^{\perp} .
$$

Proof. First suppose that the distribution $D^{\perp}$ is integrable. Then $[Y, Z] \in D^{\perp}$ for any $Y, Z \in D^{\perp}$. Since $P$ is a projection operator on $D$, so $P[Y, Z]=0$. Thus from (5.15) we get (5.20). Conversely, we suppose that (5.20) holds. Then using (5.15), we have $\phi P[Y, Z]=0$ for any $Y, Z \in D^{\perp}$. Since $\operatorname{rank} \phi=2 n$. Therefore, either $P[Y, Z]=0$ or $P[Y, Z]=k \xi$. But $P[Y, Z]=k \xi$ is not possible as $P$ is a projection operator on $D$. Thus, $P[Y, Z]=0$, which is equivalent to $[Y, Z] \in D^{\perp}$ for any $Y, Z \in D^{\perp}$ and hence $D^{\perp}$ is integrable.

COROLLARY 5.5. Let $M$ be a $\xi$-horizontal CR-submanifold of a nearly trans-Sasakian manifold $\bar{M}$. Then, the distribution $D^{\perp}$ is integrable if and only if

$$
A_{\phi Y} Z-A_{\phi Z} Y=0
$$

for any $Y, Z \in D^{\perp}$.

\section{REFERENCES}

[1] A. Bejancu, CR submanifolds of a Kaehler manifold. I, Proc. Amer. Math. Soc. 69 (1978), no. $1,135-142$.

[2] A. Bejancu and N. Papaghuic, CR-submanifolds of Kenmotsu manifold, Rend. Mat. 7 (1984), no. 4, 607-622.

[3] D. E. Blair, Contact Manifolds in Riemannian Geometry, Lecture Notes in Mathematics, vol. 509, Springer-Verlag, Berlin, 1976.

[4] C. Gherghe, Harmonicity on nearly trans-Sasaki manifolds, Demonstratio Math. 33 (2000), no. 1, 151-157.

[5] D. Janssens and L. Vanhecke, Almost contact structures and curvature tensors, Kodai Math. J. 4 (1981), no. 1, 1-27.

[6] M. Kobayashi, CR submanifolds of a Sasakian manifold, Tensor (N.S.) 35 (1981), no. 3, 297-307.

[7] J. A. Oubiña, New classes of almost contact metric structures, Publ. Math. Debrecen 32 (1985), no. 3-4, 187-193.

[8] M. H. Shahid, CR-submanifolds of a trans-Sasakian manifold, Indian J. Pure Appl. Math. 22 (1991), no. 12, 1007-1012.

[9] _ CR-submanifolds of a trans-Sasakian manifold. II, Indian J. Pure Appl. Math. 25 (1994), no. 3, 299-307.

[10] M. H. Shahid, A. Sharfuddin, and S. A. Husain, CR-submanifolds of a Sasakian manifold, Univ. u Novom Sadu Zb. Rad. Prirod.-Mat. Fak. Ser. Mat. 15 (1985), no. 1, 263-278.

[11] K. Yano and M. Kon, Contact CR submanifolds, Kodai Math. J. 5 (1982), no. 2, 238-252.

Falleh R. Al-Solamy: Department of Mathematics, Faculty of Science, King Abdul AZIZ UNIVERSITY, P.O. BOX 80015, JEDDAH 21589, SAUDI ARABIA 


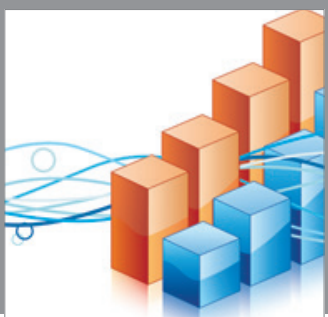

Advances in

Operations Research

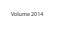

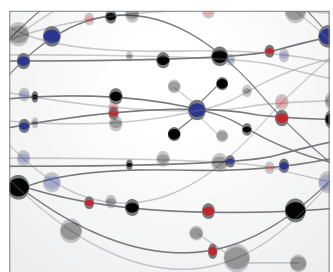

\section{The Scientific} World Journal
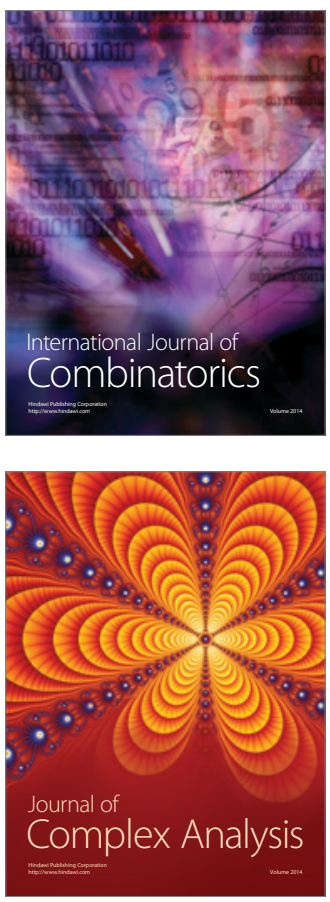

International Journal of

Mathematics and

Mathematical

Sciences
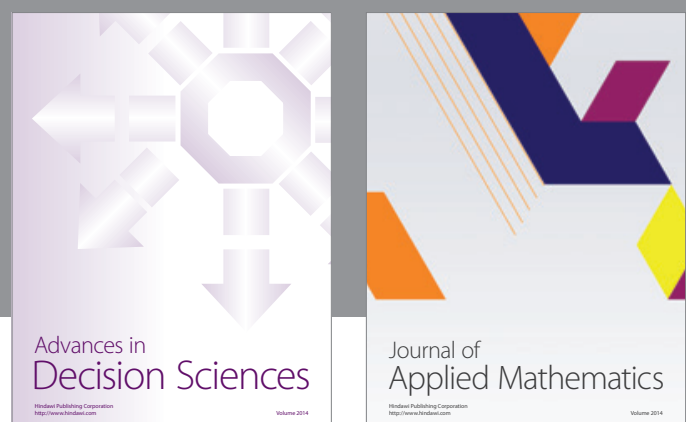

Journal of

Applied Mathematics
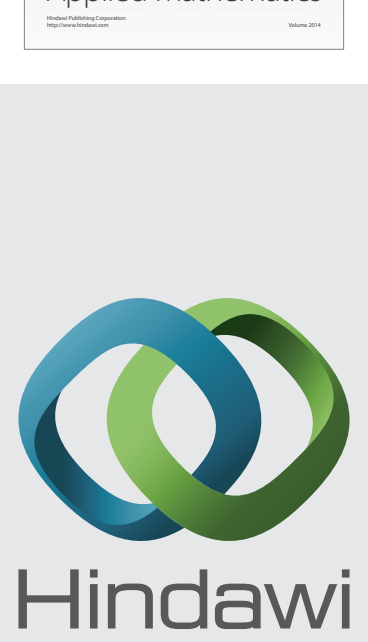

Submit your manuscripts at http://www.hindawi.com
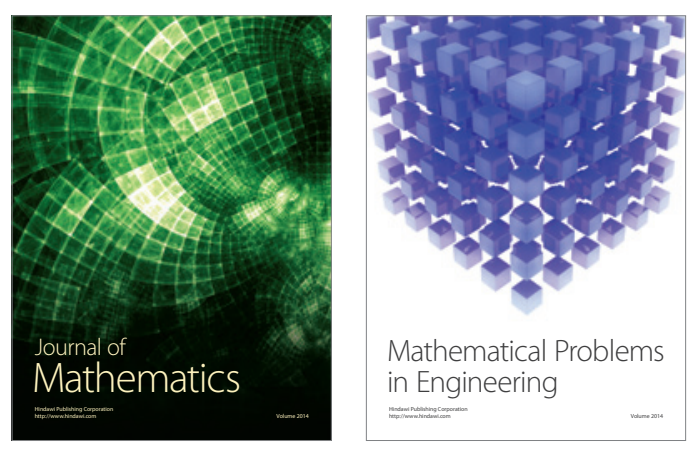

Mathematical Problems in Engineering
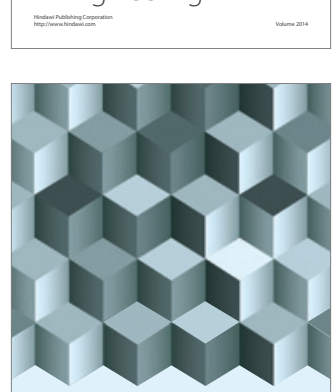

Journal of

Function Spaces
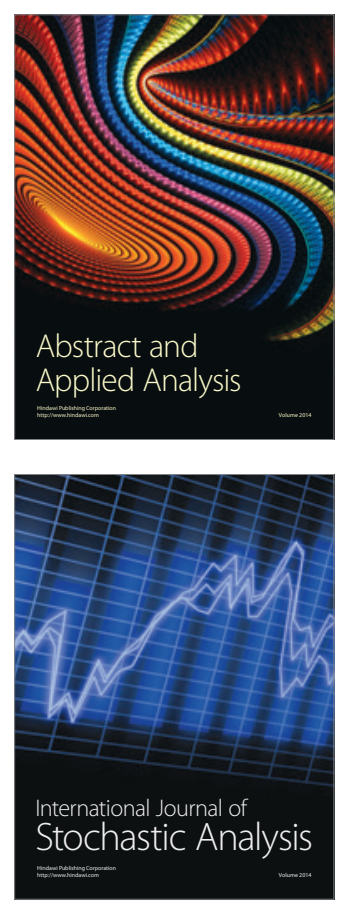

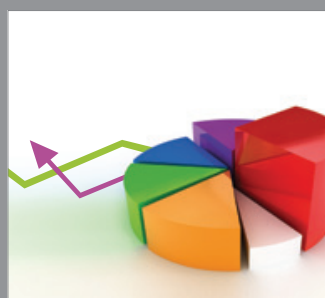

ournal of

Probability and Statistics

Promensencen
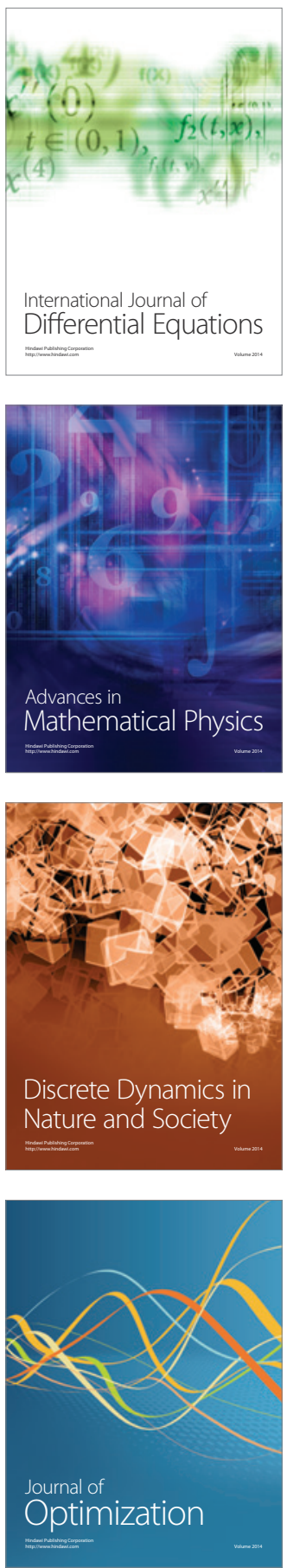